\title{
Predicting Post-operative Blood Inflammatory Biomarkers Using Pre-operative Heart Rate Variability in Patients With Cervical Cancer
}

\section{OPEN ACCESS}

Edited by:

Maurizio Schmid,

Roma Tre University, Italy

Reviewed by:

Kathleen M. Gustafson,

University of Kansas Medical Center,

United States

Kaviraja Udupa,

National Institute of Mental Health

and Neurosciences, India

*Correspondence:

Jian Liu

elitelj@126.com

Bo Shi

shibo@bbmc.edu.cn

${ }^{\dagger}$ These authors have contributed equally to this work

Specialty section:

This article was submitted to Computational Physiology and Medicine,

a section of the journal

Frontiers in Physiology

Received: 16 April 2021 Accepted: 15 October 2021 Published: 04 November 2021

Citation:

Liu J, Liu S, Gao L, Li G, Xu J, Sun Y, Wang J and Shi B (2021)

Predicting Post-operative Blood Inflammatory Biomarkers Using Pre-operative Heart Rate Variability in Patients With Cervical Cancer.

Front. Physiol. 12:696208. doi: 10.3389/fphys.2021.696208

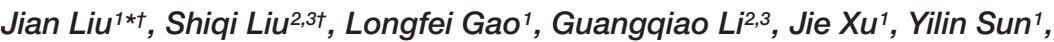 \\ Jingfeng Wang ${ }^{2,3}$ and Bo Shi2,3*
}

\begin{abstract}
' Department of Gynecologic Oncology, First Affiliated Hospital, Bengbu Medical College, Bengbu, China, ${ }^{2}$ School of Medical Imaging, Bengbu Medical College, Bengbu, China, ${ }^{3}$ Anhui Key Laboratory of Computational Medicine and Intelligent Health, Bengbu Medical College, Bengbu, China
\end{abstract}

Blood inflammatory biomarkers, including the neutrophil-to-lymphocyte ratio (NLR), the lymphocyte-to-monocyte ratio (LMR), and the platelet-to-lymphocyte ratio (PLR), play a significant role in determining the prognosis of patients with cervical cancer (CC). Currently, no methods are available to predict these indexes pre-operatively. Cardiac autonomic function is determined based on the heart rate variability (HRV), which is also associated with a progressive inflammatory response and cancer. Thus, the main aim of this study was to evaluate the feasibility of using pre-operative HRV parameters in CC patients to predict post-operative blood inflammation biomarkers as a means of determining prognosis. Between 2020 and 2021, 56 patients who were diagnosed with CC and then underwent hysterectomy surgery at the Department of Gynecologic Oncology, First Affiliated Hospital, Bengbu Medical College were enrolled in this study. Five-minute electrocardiogram data were collected 1 day before the operation for analysis of $\mathrm{HRV}$ parameters, including frequency domain parameters (LF, HF, and LF/HF) and Poincaré plot parameters (SD1, SD2, and SD2/SD1). Venous blood was collected 2 days post-operatively and inflammatory biomarkers were evaluated, with the NLR, LMR, and PLR determined. Pre-operative SD2 was significantly associated with postoperative PLR, with each 1-unit increase in SD2 decreasing the PLR value by $2.4 \pm 0.9$ $(P<0.05)$. Besides, LF/HF was significantly correlated with $N L R$, with each 1-unit increase in LF/HF increasing the NLR value by $1.1 \pm 0.5(P<0.05)$. This association was independent of patient age and body mass index. These results suggest that the pre-operative autonomic nervous system plays a role in the regulation of post-operative cancer inflammation and that pre-operative HRV parameters can potentially predict post-operative inflammation and facilitate clinical treatment decisions.

Keywords: cervical cancer, heart rate variability, autonomic nervous, inflammatory biomarkers, Poincaré plot, frequency domain

\section{INTRODUCTION}

Cervical cancer (CC) is a common gynecological malignancy, with its incidence and mortality ranked third and fourth, respectively, among female malignancies worldwide. Furthermore, CC seriously endangers a woman's physical and mental health (Bray et al., 2018). Currently, the primary treatment for CC is a radical hysterectomy and chemotherapy, with about $10 \%$ of 
patients experiencing disease recurrence (Quinn et al., 2006; Greer et al., 2010). Moreover, the high heterogeneity that is present in tumor cells often contributes to the failure of traditional cancer therapies.

With the emergence of molecular biomarkers, targeted therapy, and other therapeutic techniques, cancer treatment approaches have transformed from evidence-based medicine to precise medicine (Ho et al., 2020). However, to improve clinical precision therapy decision-making, significant prognostic information is required (Gill, 2012). Currently, cancer prognosis assessments and therapeutic effect monitoring rely on blood tests, including hematological biomarker monitoring, and pathological analyses, with post-operative pathological analysis providing a meaningful basis for prognostic evaluation that can guide further treatment (Kasamatsu et al., 2009; Holdenrieder et al., 2016; Sopik and Narod, 2018; Han et al., 2021). Nevertheless, pathological analysis is a one-time analysis and cannot be used for dynamic cancer patient monitoring. In recent years, a number of studies have confirmed that CC patient prognosis is closely associated with blood inflammatory factors. Of these hematological indicators, neutrophil-to-lymphocyte ratio (NLR), lymphocyte-to-monocyte ratio (LMR), and platelet-tolymphocyte ratio (PLR) have been shown to provide a reliable basis for evaluating $\mathrm{CC}$ patient prognosis and survival time (Trinh et al., 2020). However, post-operative blood inflammatory factors are often not predictable in advance. If the post-operative blood inflammatory factors in patients with CC can be predicted before surgery, they can provide more valuable information for the clinical decision-making of accurate treatment.

Clinical trials have demonstrated that heart rate variability (HRV), a non-invasive diagnostic tool for autonomic dysfunction, has a high specificity and positive predictive value in the prognostic assessment of cancer patients (Arab et al., 2016; Zhou et al., 2016; De Couck et al., 2018; Kloter et al., 2018). This is because autonomic nerves have a regulatory role in tumor gene expression, the surrounding microenvironment, and the inflammatory response (Cole et al., 2015; Gunterberg et al., 2016). Hu et al. (2018) demonstrated that HRV is closely associated with blood inflammatory factors, such as C-reactive protein (CRP) and interleukin (IL)-6, in patients with depression and anxiety disorders. Mouton et al. (2012) studied the relationship between HRV time-domain parameters and carcinoembryonic antigen (CEA) in 38 patients with colon cancer. They found that patients with low standard deviation of all normal-to-normal intervals (SDNN) (<20 ms) had significantly higher CEA at 1 year than patients with higher SDNN ( $\geq 20 \mathrm{~ms}$ ), independent of confounders. The study showed that low HRV was associated with increased levels of tumor markers. De Couck et al. (2013) investigated the relationship between HRV time-domain parameters and prostate-specific antigen (PSA) in 113 patients with prostate cancer. They found that SDNN and the root mean square of successive interval differences (RMSSD) were negatively correlated with PSA levels at 6 months in prostate cancer patients, controlling for numerous confounders. In addition, RMSSD was also found to be negatively correlated with PSA levels at 2 years. Thus, HRV can inversely predict the level of tumor markers in cancer patients, which has important implications for early clinical intervention and prognosis evaluation of patients. However, pre-operative HRV has not been shown to predict post-operative inflammatory factor indicators such as LMR, NLR, or PLR in CC patients. In this study, pre-operative HRV was evaluated as a potential predictive factor to elucidate these inflammatory factors in patients with CC. It is hoped this pre-operative HRV detection can potentially guide post-operative CC clinical treatment in a non-invasive manner.

\section{MATERIALS AND METHODS}

\section{Subjects}

The study cohort was comprised of CC patients $(n=60)$ who underwent hysterectomy surgery in the Department of Gynecology and Oncology, First Affiliated Hospital of Bengbu Medical College (Anhui, China) from November 2020 to January 2021. All patients met the following conditions: (i) CC diagnosed by pathological examination; (ii) treatment with hysterectomy surgery; (iii) no history of other malignant tumors; (iv) no primary diseases involving vital organs, such as heart, lung, liver, and kidney; (v) non-pregnant; (vi) no new adjuvant chemotherapy before surgery; and (vii) no missing clinical data. The study was approved by the Clinical Medical Research Ethics Committee of the First Affiliated Hospital of Bengbu Medical College (registration number: 2021KY010). All patients were informed of the details of the study, procedures, risks, and potential adverse effects of the experiment, and signed an informed consent form. The experimental procedures were performed in strict accordance with the ethical standards set forth in the 1964 Declaration of Helsinki and its amendments.

\section{Data Collection}

A HeaLink R211B micro-electrocardiogram (ECG) recorder (Healink Ltd., Bengbu, China) was used 1 day prior to surgery to collect ECG data $(5 \mathrm{~min})$ from CC patients while in a supine position. During the ECG, the test environment was kept quiet and the patients were asked to relax and breathe smoothly. Measurements were obtained with disposable $\mathrm{Ag} / \mathrm{AgCl}$ gel electrodes (JunKang Ltd., Shanghai, China). ECG signals were amplified on the device (bandwidth $0.67-40 \mathrm{~Hz}$ ) and digitized with a $400 \mathrm{~Hz}$ sampling rate.

On post-operative day 2, median cubital vein blood was collected early in the morning, and neutrophil, lymphocyte, monocyte, and platelet counts were analyzed using a Sysmex $\mathrm{XN}$ hematology analyzer (Sysmex, Kobe, Japan). Based on the findings, LMR, NLR, and PLR values were calculated.

\section{Heart Rate Variability Analysis}

The R-R interval (R-Ri) time series were extracted by ECGViewer software (HeaLink Ltd., Bengbu, China) with visual inspections afterward to remove artifacts (such as ectopic beats). Then, HRV frequency domain analysis and Poincaré plots quantitative analysis were performed. 


\section{Frequency Domain Analysis}

For the calculation of the power spectra, the R-Ri time series were resampled at $4 \mathrm{~Hz}$ using cubic spline interpolation. Subsequently, fast Fourier transform was applied. Power spectral density was calculated using Welch's periodogram method with $150 \mathrm{~s}$ window width and $50 \%$ overlapping window. The low-frequency (LF; 0.04-0.15 Hz) power, the high-frequency (HF; $0.15-0.40 \mathrm{~Hz}$ ) power, and the ratio of $\mathrm{LF}$ power to $\mathrm{HF}$ power $(\mathrm{LF} / \mathrm{HF})$ were used to evaluate HRV.

\section{Poincaré Plot Analysis}

Poincaré plot is a commonly used method in HRV non-linear analysis, which can directly show the pattern of heart rate fluctuation caused by non-linear activity. Herein, this plot was used to provide a graphical visualization of the R-Ri time series by capturing fluctuations in the intervals between heart beats (Figure 1). In this plot, each point represents an $\mathrm{R}-\mathrm{Ri}$ that is correlated with the proceeding interval, with these points distributed across a Cartesian coordinate plane (Tulppo et al., 1996). Poincaré scatter plots can also provide qualitative visual analysis and quantitative analysis. The qualitative analysis results provide supplementary information for the standard timedomain analysis of HRV signals. Quantitative analysis is based on the distribution shape of the plot to extract mathematical features, and ellipse fitting is the most conventional method for quantitative analysis. Common ellipse metrics include short-axis and long-axis measurements, with the axis along the direction of the $45^{\circ}$ contour of the ellipse being the long axis and the axis perpendicular to it being the short axis. These axes are commonly called semi-short (SD1) and semi-long (SD2) axes, where SD1 and SD2 are related to short-term and longer-term variability,

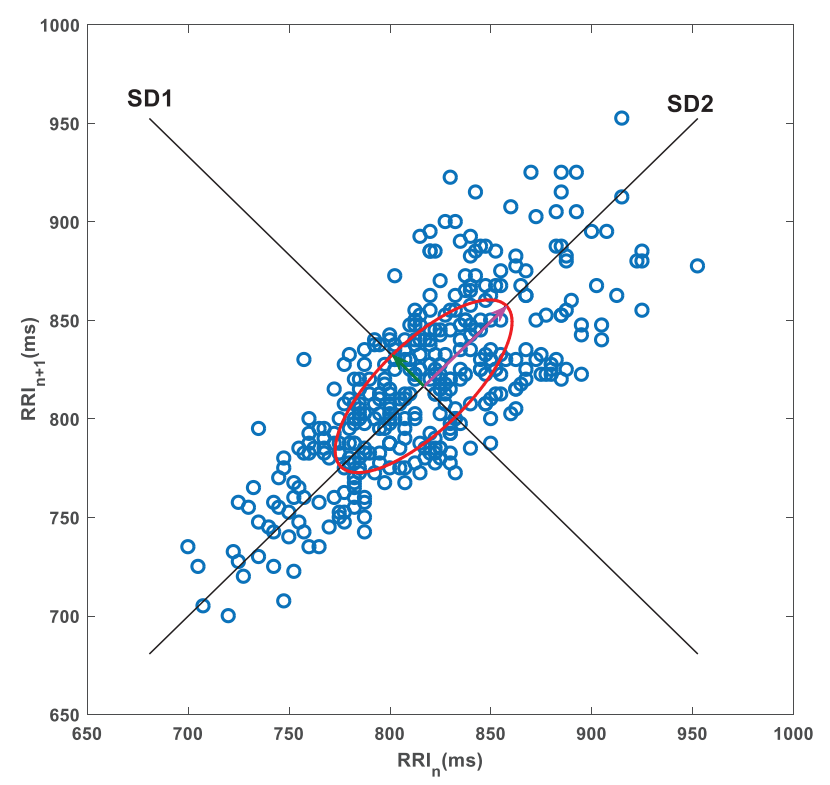

FIGURE 1 | A representative Poincaré plot. The data are consecutive RRls from a 5-min resting ECG recording from a CC patient. SD1, short axis of the ellipse; SD2, long axis of the ellipse.
TABLE 1 | Demographic, blood biomarker, and HRV CC patient data.

\begin{tabular}{lc}
\hline Variables & Values \\
\hline$N$ (Female) & 56 \\
Age (years) & $51.1(10.6)$ \\
$\mathrm{BMl}\left(\mathrm{kg} / \mathrm{m}^{2}\right)$ & $24.2(3.1)$ \\
$\mathrm{LMR}$ & $1.7(0.8)$ \\
$\mathrm{NLR}$ & $11.2(5.0)$ \\
$\mathrm{PLR}$ & $218.8(85.5)$ \\
$\mathrm{LF}\left(\mathrm{ms}^{2}\right)$ & $122(88)$ \\
$\mathrm{HF}\left(\mathrm{ms}^{2}\right)$ & $176(209)$ \\
$\mathrm{LF} / \mathrm{HF}$ & $1.468(1.284)$ \\
$\mathrm{SD} 1(\mathrm{~ms})$ & $12.4(6.7)$ \\
$\mathrm{SD} 2(\mathrm{~ms})$ & $34.5(12.2)$ \\
$\mathrm{SD} 2 / \mathrm{SD} 1$ & $3.3(1.5)$
\end{tabular}

Values are expressed as the number of patients, mean (standard deviation). Abbreviations: LF, low-frequency power; $H F$, high-frequency power; $L F / H F$, the ratio of low-frequency power to high-frequency power; NLR, the neutrophil-tolymphocyte ratio; LMR, the lymphocyte-to-monocyte ratio; PLR, the platelet-tolymphocyte ratio.

TABLE 2 | Bivariate correlation between HRV parameters and CC blood biomarkers.

\begin{tabular}{lccc}
\hline & LMR & NLR & PLR \\
\hline LF & $(0.068,0.620)$ & $(0.056,0.683)$ & $(-0.192,0.156)$ \\
HF & $(0.184,0.175)$ & $(-0.181,0.182)$ & $\mathbf{( - 0 . 2 5 2 , 0 . 0 6 1 )}$ \\
LF/HF & $(-0.165,0.223)$ & $\mathbf{( 0 . 3 0 5 , ~ 0 . 0 2 2 )}$ & $\mathbf{( 0 . 2 8 2 , 0 . 0 3 6 )}$ \\
SD1 & $(\mathbf{0 . 2 3 8 , 0 . 0 7 7 )}$ & $(-0.165,0.225)$ & $\mathbf{( - 0 . 2 3 9 , 0 . 0 7 7 )}$ \\
SD2 & $(0.147,0.279)$ & $(-0.041,0.762)$ & $\mathbf{( - 0 . 3 5 2 , 0 . 0 0 8 )}$ \\
SD2/SD1 & $(-0.212,0.117)$ & $\mathbf{( 0 . 2 3 4 , 0 . 0 8 2 )}$ & $(0.082,0.549)$ \\
\hline
\end{tabular}

Values are expressed as $(r, P)$. Bold indicates statistically significant at $P<0.1$. Abbreviations: LF, low-frequency power; HF, high-frequency power; LF/HF, the ratio of low-frequency power to high-frequency power; $N L R$, the neutrophil-tolymphocyte ratio; $L M R$, the lymphocyte-to-monocyte ratio; $P L R$, the platelet-tolymphocyte ratio.

TABLE 3 | Results from the linear regression models (adjusted for age and BMI).

\begin{tabular}{|c|c|c|c|}
\hline Predictor & Outcome & Coefficient (Estimate \pm SE) & $P$ \\
\hline SD2 & PLR & $-2.4 \pm 0.9$ & 0.009 \\
\hline LF/HF & NLR & $1.1 \pm 0.5$ & 0.036 \\
\hline SD1 & LMR & $0.04 \pm 0.02$ & 0.054 \\
\hline LF/HF & PLR & $15.5 \pm 9.3$ & 0.101 \\
\hline SD1 & PLR & $-2.3 \pm 1.8$ & 0.200 \\
\hline SD2/SD1 & NLR & $0.6 \pm 0.5$ & 0.203 \\
\hline $\mathrm{HF}$ & PLR & $-17.2 \pm 14.7$ & 0.246 \\
\hline
\end{tabular}

Bold indicates statistically significant at $P<0.05$. Abbreviations: HF, high-frequency power; $L F / H F$, the ratio of low-frequency power to high-frequency power; NLR, the neutrophil-to-lymphocyte ratio; $L M R$, the lymphocyte-to-monocyte ratio; PLR, the platelet-to-lymphocyte ratio.

respectively (Wei et al., 2020). The ratio of SD2 to SD1 (SD2/SD1) was considered as well.

\section{Statistical Analysis}

The HF histogram showed a significant right skewed distribution; therefore, the HF was log transformed prior 
to analysis. A bivariate Pearson correlation analysis was used to determine potential correlations between the HRV parameters and the blood inflammatory markers. Conservatively, features with a $P$ level of $<0.1$ were considered significant. A multiple linear regression analysis was then used to eliminate the effects of age and body mass index (BMI) with $P<0.05$ deemed significant. All statistical analyses were performed using SPSS (ver. 23.0, IBM Corp., United States).

\section{RESULTS}

Cervical cancer cases $(n=60)$ were collected, with several cases excluded due to a lack of ECG data $(n=1)$, an excessive abnormal heart beat $(n=1)$, or due to abnormal data extremes $(n=2)$. The remaining CC cases $(n=56)$ were included in this study (age: $51.1 \pm 10.6$ years; BMI: $24.2 \pm 3.1 \mathrm{~kg} / \mathrm{m}^{2}$ ), and basic information and clinical data were obtained (Table 1). A bivariate Pearson's correlation analysis was performed (Table 2), with PLR found to be positively correlated with LF/HF and negatively correlated with SD1, SD2, and HF $(P<0.05)$. Furthermore, NLR was positively correlated with both $\mathrm{LF} / \mathrm{HF}$ and SD2/SD1 $(P<0.05)$, and LMR was positively correlated with SD1 $(P<0.05)$.

To eliminate the confounding effects of age and BMI confounders, an additional multiple linear regression model was generated to eliminate these factors. The results showed that SD2 remained significantly correlated with PLR, with each 1unit increase in SD2 decreasing the PLR value by $2.4 \pm 0.9$ $(P<0.05)$. Besides, $\mathrm{LF} / \mathrm{HF}$ remained significantly correlated with NLR, with each 1-unit increase in LF/HF increasing the NLR value by $1.1 \pm 0.5(P<0.05)$ (Table 3$)$. To further examine this correlation, a partial correlation plot examining the outcome (PLR and NLR) and HRV parameters were generated (Figure 2).

\section{DISCUSSION}

The primary aim of this study was to determine if the preoperative HRV parameters are correlated with post-operative blood inflammatory biomarkers (LMR, NLR, and PLR) in $\mathrm{CC}$ patients. The findings identified a negative correlation between the pre-operative SD2 and post-operative PLR, a positive correlation between the pre-operative LF/HF and postoperative NLR, and this association persisted after excluding both age and BMI following a multiple linear regression analysis. Overall, these results indicate that the use of pre-operative HRV to predict post-operative blood inflammatory markers in CC patients is promising.

Many studies have confirmed that autonomic nervous regulation is associated with the proliferation of inflammatory cells, including neutrophils, lymphocytes, and monocytes (Abo and Kawamura, 2002). Neutrophils and lymphocytes have adrenaline and acetylcholine receptors on their surfaces, which are regulated by both sympathetic and parasympathetic nerves. Specifically, when the sympathetic system is activated, the number of neutrophils increases, the number of lymphocytes decreases, and the value of NLR increases (Cao and Lawrence, 2002; Engler et al., 2004; Emeny et al., 2007). Conversely, when the parasympathetic nerve is excited, the number of neutrophils decreases, the number of lymphocytes increases, and the value of NLR decreases (Ono and Okada, 2013). LF/HF can be used to quantify the relationship between sympathetic and parasympathetic activity (Task Force of the European Society of Cardiology and the North American Society of Pacing and Electrophysiology, 1996). The increase in LF/HF is thought to reflect a shift to "sympathetic dominance," while the decline in this index corresponds to "parasympathetic dominance." The positive correlation between $\mathrm{LF} / \mathrm{HF}$ and post-operative NLR in our study confirmed the regulatory effect of autonomic nerve on post-operative inflammation.

Platelet-to-lymphocyte ratio is considered a novel biomarker that reflects the inflammatory response and immune state of the body, as well as being closely related to the activation of the coagulation system (Zhao et al., 2018). SD2 quantitatively reflects the overall degree of heart rate variation, which represents the shared role of the sympathetic and vagus nerves (Shaffer and Ginsberg, 2017). Sympathetic activation promotes lymphocytopenia and can also promote bone marrow megakaryocyte proliferation, which subsequently
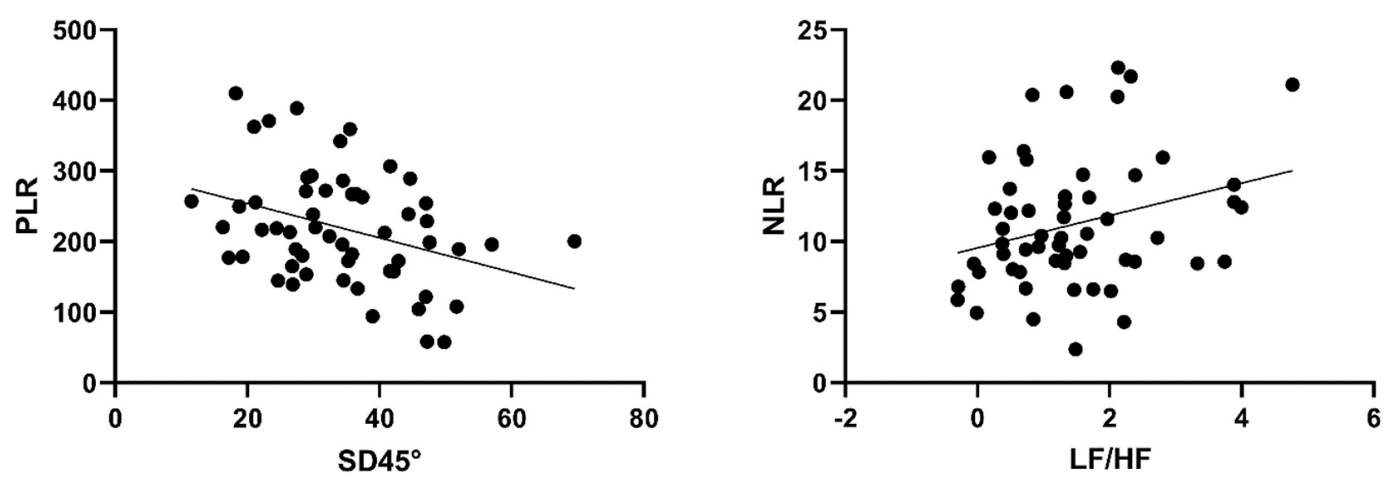

FIGURE 2 | Partial correlation plot for HRV parameters and blood biomarkers after correcting for age and BMI. 
increases the number of circulating platelets and the rate of platelet aggregation ( $\mathrm{Fu}$ et al., 2019). These previous findings in conjunction with the physiological findings presented herein suggest the overall HRV mediated by sympathetic and vagus nerves has a negative association with the ratio of platelets to lymphocytes. However, the long-term effect of this association between the autonomic function and platelets is still unclear and will require further examination.

Current studies on multiple malignancies, including colorectal cancer (CRC), hepatocellular carcinoma (HCC), non-small cell lung cancer (NSCLC), ovarian cancer (OC), and CC, have shown that a high PLR is associated with a poor prognosis in cancer patients (Asher et al., 2011; Kwon et al., 2012). In CC studies, PLR has been shown to be significantly correlated with lymph node association and distant metastasis, as well as the response to radiotherapy (Zhang et al., 2014). Additionally, PLR can also be used as an independent International Federation of Gynecology and Obstetrics (FIGO) stage predictor (Zhu et al., 2018; Tas et al., 2019). Moreover, Tas et al. (2019) found that patients with invasive CC had a higher PLR when compared to a low-grade squamous intraepithelial lesion (LSIL). By contrast, a higher PLR is seen in high-grade squamous intraepithelial lesions (HSILs) when compared to patients with invasive CC, with elevated PLR and NLR both predictive of CC. In cancer patients, HRV has been shown to be significantly lower relative to healthy individuals, with levels also significantly lower in metastatic patients or patients with advanced stage III or IV when compared to non-metastatic patients (Bettermann et al., 2001; Nevruz et al., 2007; Lin and Chen, 2010; Koszewicz et al., 2016; Palma et al., 2016). Furthermore, in a study examining tumor markers in CRC patients, tumor marker levels were significantly increased in patients with a low HRV (Mouton et al., 2012). Wang et al. (2013) found that patients with brain metastases had significantly lower SDNN values relative to healthy individuals, with patients with an SDNN $<10 \mathrm{~ms}$ having a significantly lower survival than those with an SDNN > $10 \mathrm{~ms}$. In another study investigating the relationship between HRV time-domain parameters and survival in pancreatic cancer, a higher SDNN (>20 ms) was significantly associated with a longer survival independent of confounding factors such as age or cancer treatment (De Couck et al., 2016). Previous studies have also shown that a high PLR and low HRV are indicative of a poor cancer prognosis, which is consistent with the findings presented herein.

At present, PLR, NLR, LMR, and other indicators can only be detected using invasive methods and cannot be predicted. In contrast, HRV analysis provides a noninvasive tool for assessing autonomic nervous system activity, usually by analyzing ECG time intervals or pulse wave signals (Kloter et al., 2018). Thus, the correlation between pre-operative HRV parameters and post-operative blood inflammatory factors identified herein tentatively provides a means to predict the prognosis of a CC patient pre-operatively and subsequently aid in guiding clinical treatment decisions.
Based on the above discussion, our study confirmed that it is feasible to predict post-operative blood inflammatory factors by examining pre-operative HRV values. However, this study also has some limitations. First, a previous study suggested that immune cell proportions and numbers follow a circadian rhythm and appear to be regulated by the autonomic nervous system (Suzuki et al., 1997). For example, during the day, the sympathetic nerve is active and the neutrophil numbers are increased. By contrast, at night, the vagus nerve is dominant and the lymphocyte numbers are increased. In this study, the samples were only collected in the morning; thus, different times of day could be optimal, and therefore other times should be explored in future work. Second, the potential long-term effects of autonomic function may lead to inflammatory level changes over time, which will require further investigating. Moving forward, it is necessary to study the dynamic fluctuations of HRV and inflammatory markers at different time points before and after surgery to understand the changes of autonomic nerve modulation over time during the inflammation process. Additionally, future studies should focus on examining a larger sample size and should consider more confounding factors.

\section{DATA AVAILABILITY STATEMENT}

The raw data supporting the conclusions of this article will be made available by the authors, without undue reservation.

\section{ETHICS STATEMENT}

The study was approved by the Clinical Medical Research Ethics Committee of the First Affiliated Hospital of Bengbu Medical College (Registration Number: 2021KY010). The patients/participants provided their written informed consent to participate in this study.

\section{AUTHOR CONTRIBUTIONS}

BS and JL: conceptualization and resources. GL, JX, and YS: data collection. All authors: methodology. GL and JW: formal analysis. JL: data curation and supervision. BS and SL: writing-original draft preparation. BS, SL, and JL: writing-review and editing. BS: project administration and funding acquisition. All authors contributed to the article and approved the submitted version.

\section{FUNDING}

This research was funded by the "512" Outstanding Talents Fostering Project of Bengbu Medical College (Grant Number BY51201312) and the Scientific Research Innovation Project of Bengbu Medical College (Grant Number BYKC201905). 


\section{REFERENCES}

Abo, T., and Kawamura, T. (2002). Immunomodulation by the autonomic nervous system: therapeutic approach for cancer, collagen diseases, and inflammatory bowel diseases. Ther. Apher. 6, 348-357. doi: 10.1046/j.1526-0968.2002.00452.x

Arab, C., Dias, D. P., Barbosa, R. T., Carvalho, T. D., Valenti, V. E., Crocetta, T. B., et al. (2016). Heart rate variability measure in breast cancer patients and survivors: a systematic review. Psychoneuroendocrinology 68, 57-68. doi: 10.1016/j.psyneuen.2016.02.018

Asher, V., Lee, J., Innamaa, A., and Bali, A. (2011). Preoperative platelet lymphocyte ratio as an independent prognostic marker in ovarian cancer. Clin. Transl. Oncol. 13, 499-503. doi: 10.1007/s12094-011-0687-9

Bettermann, H., Kröz, M., Girke, M., and Heckmann, C. (2001). Heart rate dynamics and cardiorespiratory coordination in diabetic and breast cancer patients. Clin. Physiol. 21, 411-420. doi: 10.1046/j.1365-2281.2001.00342.x

Bray, F., Ferlay, J., Soerjomataram, I., Siegel, R. L., Torre, L. A., and Jemal, A. (2018). Global cancer statistics 2018: GLOBOCAN estimates of incidence and mortality worldwide for 36 cancers in 185 countries. CA Cancer J. Clin. 68, 394-424. doi: 10.3322/caac. 21492

Cao, L., and Lawrence, D. A. (2002). Suppression of host resistance to Listeria monocytogenes by acute cold/restraint stress: lack of direct IL-6 involvement. J. Neuroimmunol. 133, 132-143. doi: 10.1016/s0165-5728(02)00371-5

Cole, S. W., Nagaraja, A. S., Lutgendorf, S. K., Green, P. A., and Sood, A. K. (2015). Sympathetic nervous system regulation of the tumour microenvironment. Nat. Rev. Cancer 15, 563-572. doi: 10.1038/nrc3978

De Couck, M., Caers, R., Spiegel, D., and Gidron, Y. (2018). The role of the vagus nerve in cancer prognosis: a systematic and a comprehensive review. J. Oncol. 2018:1236787. doi: 10.1155/2018/1236787

De Couck, M., Maréchal, R., Moorthamers, S., Van Laethem, J. L., and Gidron, Y. (2016). Vagal nerve activity predicts overall survival in metastatic pancreatic cancer, mediated by inflammation. Cancer Epidemiol. 40, 47-51. doi: 10.1016/ j.canep.2015.11.007

De Couck, M., van Brummelen, D., Schallier, D., De Grève, J., and Gidron, Y. (2013). The relationship between vagal nerve activity and clinical outcomes in prostate and non-small cell lung cancer patients. Oncol. Rep. 30, 2435-2441. doi: $10.3892 /$ or. 2013.2725

Emeny, R. T., Gao, D., and Lawrence, D. A. (2007). Betal-adrenergic receptors on immune cells impair innate defenses against Listeria. J. Immunol. 178, 4876-4884. doi: 10.4049/jimmunol.178.8.4876

Engler, H., Dawils, L., Hoves, S., Kurth, S., Stevenson, J. R., Schauenstein, K., et al. (2004). Effects of social stress on blood leukocyte distribution: the role of alpha- and beta-adrenergic mechanisms. J. Neuroimmunol. 156, 153-162. doi: 10.1016/j.jneuroim.2004.08.005

Fu, W., Wang, J., Jiang, H., and Hu, X. (2019). Myocardial infarction induces bone marrow megakaryocyte proliferation, maturation and platelet production. Biochem. Biophys. Res. Commun. 510, 456-461. doi: 10.1016/j.bbrc.2019.0 1.129

Gill, T. M. (2012). The central role of prognosis in clinical decision making. JAMA 307, 199-200. doi: 10.1001/jama.2011.1992

Greer, B. E., Koh, W. J., Abu-Rustum, N. R., Apte, S. M., Campos, S. M., Chan, J., et al. (2010). Cervical cancer. J. Natl. Compr. Canc. Netw. 8, 1388-1416. doi: 10.6004/jnccn.2010.0104

Gunterberg, V., Simrén, M., Öhman, L., Friberg, P., Jones, M. P., Van Oudenhove, L., et al. (2016). Autonomic nervous system function predicts the inflammatory response over three years in newly diagnosed ulcerative colitis patients. Neurogastroenterol. Motil. 28, 1655-1662. doi: 10.1111/nmo.12865

Han, X., Liu, S., Yang, G., Hosseinifard, H., Imani, S., Yang, L., et al. (2021). Prognostic value of systemic hemato-immunological indices in uterine cervical cancer: a systemic review, meta-analysis, and meta-regression of observational studies. Gynecol. Oncol. 160, 351-360. doi: 10.1016/j.ygyno.2020.10.011

Ho, D., Quake, S. R., McCabe, E. R. B., Chng, W. J., Chow, E. K., Ding, X., et al. (2020). Enabling technologies for personalized and precision medicine. Trends Biotechnol. 38, 497-518. doi: 10.1016/j.tibtech.2019.12.021

Holdenrieder, S., Pagliaro, L., Morgenstern, D., and Dayyani, F. (2016). Clinically meaningful use of blood tumor markers in oncology. Biomed. Res. Int. 2016:9795269. doi: 10.1155/2016/9795269

Hu, M. X., Lamers, F., Neijts, M., Willemsen, G., de Geus, E. J. C., and Penninx, B. W. J. H. (2018). Bidirectional prospective associations between cardiac autonomic activity and inflammatory markers. Psychosom. Med. 80, 475-482. doi: 10.1097/PSY.0000000000000589

Kasamatsu, T., Onda, T., Sawada, M., Kato, T., and Ikeda, S. (2009). Radical hysterectomy for FIGO stage IIB cervical cancer: clinicopathological characteristics and prognostic evaluation. Gynecol. Oncol. 114, 69-74. doi: 10.1016/j.ygyno.2009.03.026

Kloter, E., Barrueto, K., Klein, S. D., Scholkmann, F., and Wolf, U. (2018). Heart rate variability as a prognostic factor for cancer survival - a systematic review. Front. Physiol. 9:623. doi: 10.3389/fphys.2018.00623

Koszewicz, M., Michalak, S., Bilinska, M., Budrewicz, S., Zaborowski, M., Slotwinski, K., et al. (2016). Profile of autonomic dysfunctions in patients with primary brain tumor and possible autoimmunity. Clin. Neurol. Neurosurg. 151, 51-54. doi: 10.1016/j.clineuro.2016.10.013

Kwon, H. C., Kim, S. H., Oh, S. Y., Lee, S., Lee, J. H., Choi, H. J., et al. (2012) Clinical significance of preoperative neutrophil-lymphocyte versus plateletlymphocyte ratio in patients with operable colorectal cancer. Biomarkers 17, 216-222. doi: 10.3109/1354750X.2012.656705

Lin, S. C., and Chen, M. F. (2010). Increased yin-deficient symptoms and aggravated autonomic nervous system function in patients with metastatic cancer. J. Altern. Complement. Med. 16, 1059-1063. doi: 10.1089/acm.2009. 0487

Mouton, C., Ronson, A., Razavi, D., Delhaye, F., Kupper, N., Paesmans, M., et al. (2012). The relationship between heart rate variability and time-course of carcinoembryonic antigen in colorectal cancer. Auton. Neurosci. 166, 96-99. doi: 10.1016/j.autneu.2011.10.002

Nevruz, O., Yokusoglu, M., Uzun, M., Demirkol, S., Avcu, F., Baysan, O., et al. (2007). Cardiac autonomic functions are altered in patients with acute leukemia, assessed by heart rate variability. Tohoku J. Exp. Med. 211, 121-126. doi: $10.1620 /$ tjem.211.121

Ono, M., and Okada, S. (2013). Effect of acetylcholine on mitogen response of peripheral lymphocytes isolated from rats exposed to chronic stress. Biol. Res. Nurs. 15, 71-77. doi: 10.1177/1099800411415663

Palma, M. R., Vanderlei, L. C., Ribeiro, F. E., Mantovani, A. M., Christofaro, D. G., and Fregonesi, C. E. (2016). The relationship between post-operative time and cardiac autonomic modulation in breast cancer survivors. Int. J. Cardiol. 224, 360-365. doi: 10.1016/j.ijcard.2016.09.053

Quinn, M. A., Benedet, J. L., Odicino, F., Maisonneuve, P., Beller, U., Creasman, W. T., et al. (2006). Carcinoma of the cervix uteri. FIGO 26th annual report on the results of treatment in gynecological cancer. Int. J. Gynaecol. Obstet. 95, S43-S103. doi: 10.1016/S0020-7292(06)60030-1

Shaffer, F., and Ginsberg, J. P. (2017). An Overview of Heart Rate Variability Metrics and Norms. Front. Public Health 5:258. doi: 10.3389/fpubh.2017.00258

Sopik, V., and Narod, S. A. (2018). The relationship between tumour size, nodal status and distant metastases: on the origins of breast cancer. Breast Cancer Res. Treat. 170, 647-656. doi: 10.1007/s10549-018-4796-9

Suzuki, S., Toyabe, S., Moroda, T., Tada, T., Tsukahara, A., Iiai, T., et al. (1997). Circadian rhythm of leucocytes and lymphocytes subsets and its possible correlation with the function of the autonomic nervous system. Clin. Exp. Immunol. 110, 500-508. doi: 10.1046/j.1365-2249.1997.4411460.x

Tas, M., Yavuz, A., Ak, M., and Ozcelik, B. (2019). Neutrophil-to-lymphocyte ratio and platelet-to-lymphocyte ratio in discriminating precancerous pathologies from cervical cancer. J. Oncol. 2019:2476082. doi: 10.1155/2019/2476082

Task Force of the European Society of Cardiology and the North American Society of Pacing and Electrophysiology (1996). Heart rate variability: standards of measurement, physiological interpretation and clinical use. Circulation 93, 1043-1065. doi: 10.1161/01.cir.93.5.1043

Trinh, H., Dzul, S. P., Hyder, J., Jang, H., Kim, S., Flowers, J., et al. (2020). Prognostic value of changes in neutrophil-to-lymphocyte ratio (NLR), plateletto-lymphocyte ratio (PLR) and lymphocyte-to-monocyte ratio (LMR) for patients with cervical cancer undergoing definitive chemoradiotherapy (dCRT). Clin. Chim. Acta 510, 711-716. doi: 10.1016/j.cca.2020.09.008

Tulppo, M. P., Mäkikallio, T. H., Takala, T. E., Seppänen, T., and Huikuri, H. V. (1996). Quantitative beat-to-beat analysis of heart rate dynamics during exercise. Am. J. Physiol. 271, H244-H252. doi: 10.1152/ajpheart.1996.271.1. $\mathrm{H} 244$

Wang, Y. M., Wu, H. T., Huang, E. Y., Kou, Y. R., and Hseu, S. S. (2013). Heart rate variability is associated with survival in patients with brain metastasis: a preliminary report. Biomed Res. Int. 2013:503421. doi: 10.1155/2013/503421 
Wei, Y., Liang, Y., Lin, H., Dai, Y., and Yao, S. (2020). Autonomic nervous system and inflammation interaction in endometriosis-associated pain. J. Neuroinflammation 17:80. doi: 10.1186/s12974-020-01752-1

Zhang, Y., Wang, L., Liu, Y., Wang, S., Shang, P., Gao, Y., et al. (2014). Preoperative neutrophil-lymphocyte ratio before platelet-lymphocyte ratio predicts clinical outcome in patients with cervical cancer treated with initial radical surgery. Int. J. Gynecol. Cancer 24, 1319-1325. doi: 10.1097/IGC.0000000000000219

Zhao, Z., Zhao, X., Lu, J., Xue, J., Liu, P., and Mao, H. (2018). Prognostic roles of neutrophil to lymphocyte ratio and platelet to lymphocyte ratio in ovarian cancer: a meta-analysis of retrospective studies. Arch. Gynecol. Obstet. 297, 849-857. doi: 10.1007/s00404-018-4678-8

Zhou, X., Ma, Z., Zhang, L., Zhou, S., Wang, J., Wang, B., et al. (2016). Heart rate variability in the prediction of survival in patients with cancer: a systematic review and meta-analysis. J. Psychosom. Res. 89, 20-25. doi: 10.1016/ j.jpsychores.2016.08.004

Zhu, M., Feng, M., He, F., Han, B., Ma, K., Zeng, X., et al. (2018). Pretreatment neutrophil-lymphocyte and platelet-lymphocyte ratio predict clinical outcome and prognosis for cervical Cancer. Clin. Chim. Acta 483, 296-302. doi: 10.1016/ j.cca.2018.05.025
Conflict of Interest: A direct family member of BS owns stock in HeaLink Ltd., Bengbu, China.

The remaining authors declare that the research was conducted in the absence of any commercial or financial relationships that could be construed as a potential conflict of interest.

Publisher's Note: All claims expressed in this article are solely those of the authors and do not necessarily represent those of their affiliated organizations, or those of the publisher, the editors and the reviewers. Any product that may be evaluated in this article, or claim that may be made by its manufacturer, is not guaranteed or endorsed by the publisher.

Copyright (c) $2021 \mathrm{Liu}, \mathrm{Liu}, \mathrm{Gao}, \mathrm{Li}, \mathrm{Xu}$, Sun, Wang and Shi. This is an open-access article distributed under the terms of the Creative Commons Attribution License (CC BY). The use, distribution or reproduction in other forums is permitted, provided the original author(s) and the copyright owner(s) are credited and that the original publication in this journal is cited, in accordance with accepted academic practice. No use, distribution or reproduction is permitted which does not comply with these terms. 